\title{
Bariatric surgery in obese patients with ventricular assist devices
}

\author{
Adrian daSilva-deAbreu ${ }^{1,2,3^{*}}$ (D) Bader Aldeen Alhafez ${ }^{4}$, Yuhamy Curbelo-Pena ${ }^{5}$, Carl J. Lavie ${ }^{1,2}$, \\ Hector O. Ventura ${ }^{1,2}$, Juan Francisco Loro-Ferrer ${ }^{3}$ and Stacy A. Mandras ${ }^{1,2}$
}

\begin{abstract}
Objectives: Patients with end-stage heart failure (ESHF) treated with ventricular assist devices (VADs) tend to gain weight, which may prevent them from receiving heart transplantation (HT) if their body mass index (BMI) reaches $\geq 35 \mathrm{~kg} / \mathrm{m}^{2}$. The objective was to synthesize all cases available in the literature and describe the most important outcomes of bariatric surgery (BS) in VAD patients, including BMl trends, reaching a BMl $<35 \mathrm{~kg} / \mathrm{m}^{2}$, listing for $H T$, achieving HT, myocardial recovery, and mortality. These data were obtained for an individual participant data (IPD) meta-analysis and include available IPD for every case in the scientific literature describing VAD patients undergoing BS during VAD support with documented postoperative BMI (and time of measurement) during follow-up.

Data description: These data include baseline, periprocedural, and long-term outcomes for the 29 patients meeting selection criteria. The composite outcome includes reaching a $\mathrm{BMl}<35 \mathrm{~kg} / \mathrm{m}^{2}$, listing for $\mathrm{HT}$, receiving $\mathrm{HT}$, and myocardial recovery, indicating significant BMI loss associated with major ESHF outcomes. As multiple centers are becoming more experienced in this field, the present data can be merged with their databases to form larger samples that will allow to perform further statistical analysis to identify outcome predictors and improve clinical protocols and outcomes.
\end{abstract}

Keywords: Heart-assist devices, Ventricular assist devices, Bariatric surgery, Sleeve gastrectomy, Gastric bypass, Rouxen-Y gastric bypass, Obesity, Heart failure, Body mass index, Heart transplantation

\section{Objective}

Patients with class II obesity (body mass index; $\mathrm{BMI} \geq 35 \mathrm{~kg} / \mathrm{m}^{2}$ ) have worse outcomes after heart transplantation (HT). Hence, the International Society for Heart and Lung Transplantation considers a BMI $\geq 35 \mathrm{~kg} /$ $\mathrm{m}^{2}$ to be a contraindication for HT [1], excluding many obese patients with end-stage heart failure (ESHF) from transplantation, which is the gold standard therapy for this condition.

An alternative therapy for obese patients with ESHF is ventricular assist device (VAD) implantation. The BMI cutoff for VAD implantation varies among

\footnotetext{
*Correspondence: adrianjdasilva@gmail.com

1 John Ochsner Heart and Vascular Institute, Ochsner Clinic Foundation, New Orleans, LA, USA

Full list of author information is available at the end of the article
}

different centers and is often higher than for HT. Based on this, one would expect obese patients to undergo VAD implantation, lose weight, and undergo HT once they achieve a $\mathrm{BMI}<35 \mathrm{~kg} / \mathrm{m}^{2}$. However, patients with BMIs $<35 \mathrm{~kg} / \mathrm{m}^{2}$ tend to gain weight after VAD implantation [2], which may halt their candidacy for HT if they reach a $B M I \geq 35 \mathrm{~kg} / \mathrm{m}^{2}$.

Few small cohort studies and case reports have shown promising results of bariatric surgery (BS) in obese patients with VADs [3-6]. Nevertheless, much of the postoperative weight (and BMI) trends remains unknown in this population. This is an important knowledge gap because VAD patients receive chronic anticoagulation and are already at very high risk of thromboembolic and bleeding events at baseline [7] which increases the risk of adverse events after surgeries. In consequence, only few

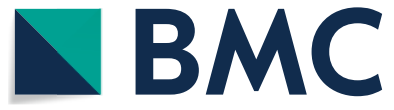

(c) The Author(s) 2020. This article is licensed under a Creative Commons Attribution 4.0 International License, which permits use, sharing, adaptation, distribution and reproduction in any medium or format, as long as you give appropriate credit to the original author(s) and the source, provide a link to the Creative Commons licence, and indicate if changes were made. The images or other third party material in this article are included in the article's Creative Commons licence, unless indicated otherwise in a credit line to the material. If material is not included in the article's Creative Commons licence and your intended use is not permitted by statutory regulation or exceeds the permitted use, you will need to obtain permission directly from the copyright holder. To view a copy of this licence, visit http://creativeco mmons.org/licenses/by/4.0/. The Creative Commons Public Domain Dedication waiver (http://creativecommons.org/publicdomain/ zero/1.0/) applies to the data made available in this article, unless otherwise stated in a credit line to the data. 
large centers offer this intervention to a minority of obese patients with VADs.

Therefore, this systematic review and individual participant data (IPD) meta-analysis included available IPD for every case in the scientific literature describing the outcomes of VAD patients who underwent BS with documented postoperative BMI (and time of measurement) during follow-up [8]. The aim was to evaluate the most important outcomes after BS in VAD patients, including BMI trends, listing for $\mathrm{HT}$, achieving $\mathrm{HT}$, myocardial recovery, and mortality.

\section{Data description}

A systematic search was conducted in PubMed, Embase, The Cochrane Library and ClinicalTrials.gov combining free keywords and official indexing terms for VADs and BS. Truncated terms were used when allowed by the engine. Additional searches were performed in Google Scholar and the online content of: The Journal of Heart and Lung Transplantation, Journal of Cardiac Failure, Obesity Surgery, Surgery for Obesity and Related Diseases, and every member of the journal groups: Journal of the American College of Cardiology, Circulation, and the European Heart Journal.

Retrieved references were transferred to EndNote X8 (Clarivate Analytics, Philadelphia, PA, USA) and Rayyan (Qatar Computing Research Institute, Doha, Qatar), and duplicates were removed. Studies of any design and publication type (abstracts, full article, registry with results...) were included if they contained IPD of postoperative BMIs of VAD patients undergoing BS. Two investigators worked in parallel and independently to select references, extract IPD, and assess the risk of bias in each study. Discrepancies were resolved by discussion.

Every study was classified according to the institution where they were conducted, publication year, sample size, number of patients that met selection criteria, publication type, and whether or not they met selection criteria, data available in Data file 1. Only non-duplicated IPD were obtained from reference(s) with largest sample size with available IPD for postoperative BMI. One patient's IPD was included in a case report and a cohort study $[9,10]$, hence, such IPD were extracted from the most detailed reference [9]. When available, weight and additional anthropometric data were used to calculate BMI. All included studies had low risk of bias for the main outcome (postoperative BMI).

Data file 2 provides detailed data of the studies and baseline characteristics of patients that met selection criteria. Detailed BMI data over time, type of BS, length of follow-up, and most important clinical ESHF outcomes are available in Data file 3. These data evidence successful results of $\mathrm{BS}$ in obese VAD patients to achieve the composite outcome of $\mathrm{a}<35 \mathrm{~kg} / \mathrm{m}^{2}, \mathrm{HT}$, listing for HT, or myocardial recovery.

Data file 4 displays graphical trends of the excess BMI lost over time after BS. Each line represents an individual patient and zero (0) corresponds to the time and baseline body mass index at the time of bariatric surgery which is the point of reference for BMI comparisons (excess BMI lost) during follow-up. This graph was developed with StataSE 14 (College Station, TX, USA). Data file 5 provides a list of the references cited in the tables.

As clinical practice and research interest in this field continues to grow, multiple centers of advanced heart failure and HT will potentially benefit from analyzing these data or merging these data with their own (single or multicenter) data to create a larger sample to perform more elaborate statistical analysis, such as regressions and subgroup analyses, to better understand the impact of BS in these obese patients with VADs and to identify predictors of major outcomes that ultimately translate into improvements in clinical practice and outcomes.

\section{Limitations}

- As with every meta-analysis, there is a risk of publication bias of the available literature which could have biased our sample. To reduce this risk, we did not exclude abstracts and screened grey literature.

- There is also a risk of reporting bias, especially with case reports that did not report all major outcomes of interest.

- These data contain IPD of patients who already had VADs at the time of BS. Patients who underwent BS prior to VAD implantation or had both interventions done during the same encounter were excluded. Patients without available postoperative BMI data were excluded because the meta-analysis was centered on the BMI trends after BS. However, these data are very valuable as they provide better understanding and could even expedite future meta-analyses in the field by greatly reducing the amount of work required for data extraction and quality control. Furthermore, identifying the missing data from each reference allows to appreciate areas for improvement in the way we report studies and gaps of knowledge on this topic. 
Table 1 Overview of data files/data sets

\begin{tabular}{|c|c|c|c|}
\hline Label & Name of data file/data set & File types (file extension) & Data repository and identifier (DOI or accession number) \\
\hline Data file 1 & $\begin{array}{l}\text { Data file 1. References of patients with VAD } \\
\text { undergoing bariatric surgery.xIsx }\end{array}$ & $x \mid s x($ or Excel) & Mendeley (https://doi.org/10.17632/33m7sc3wnc.2) \\
\hline Data file 2 & Data file 2. Baseline patient characteristics.xIsx & $x / s x($ or Excel) & Mendeley (https://doi.org/10.17632/33m7sc3wnc.2) \\
\hline Data file 3 & Data file 3. Perioperative and outcome data.x/sx & $x \mid s x($ or Excel) & Mendeley (https://doi.org/10.17632/33m7sc3wnc.2) \\
\hline Data file 4 & $\begin{array}{l}\text { Data file 4. Trends of excess BMI after bariatric } \\
\text { surgery in patients with VADs.png }\end{array}$ & png & Mendeley (https://doi.org/10.17632/33m7sc3wnc.2) \\
\hline Data file 5 & Data file 5. References for Data files 1-3.docx & docx (or Word) & Mendeley (https://doi.org/10.17632/33m7sc3wnc.2) \\
\hline
\end{tabular}

\section{Abbreviations}

BMI: Body mass index; BS: Bariatric surgery; ESHF: End-stage heart failure; HT: Heart transplantation; IPD: Individual participant data; VAD: Ventricular assist device.

\section{Acknowledgements}

This article is part of the doctoral research thesis of Adrian daSilva-deAbreu, who is the principal investigator of this study and main author of this paper. All other authors commit to not using this publication as main part of their doctoral theses. However, this does not mean that this article cannot be used by the other authors as complement merit in their doctoral theses.

\section{Author contributions}

AdS-dA, BAA, and YC-P contributed with study design, data gathering, data processing, as well as manuscript drafting and editing. AdS-dA conducted the statistical analysis. CJL, HOV, JFL-F, and SAM contributed with interpretation of results, supervision of the study, and manuscript editing. All authors read and approved the final manuscript.

\section{Funding}

None.

\section{Availability of data and materials}

The data described in this Data note can be freely and openly accessed on Mendeley Data under https://doi.org/10.17632/33m7sc3wnc.2 [11]. Please see Table 1 and references 8 and 11 for details and links to the data.

\section{Ethics approval and consent to participate}

Not applicable. This data was collected and processed from the available published literature as part of a meta-analysis, hence, available patient data from each study from each study should have been approved institutional review boards (bioethics committees) of author's institutions and/or undergone patient consent.

\section{Consent for publication}

Not applicable.

\section{Competing interests}

SAM is a paid speaker for United Therapeutics Corporation and Bayer. All other authors declare no conflicts of interest related to this study.

\section{Author details}

1 John Ochsner Heart and Vascular Institute, Ochsner Clinic Foundation, New Orleans, LA, USA. ${ }^{2}$ The University of Queensland Ochsner Clinical School, Faculty of Medicine, The University of Queensland, New Orleans, LA, USA. ${ }^{3}$ Doctoral School, Universidad de Las Palmas de Gran Canaria, Las Palmas, Spain. ${ }^{4}$ Department of Internal Medicine, The Ohio State University, Columbus, OH, USA. ${ }^{5}$ Service of General Surgery, Consorci Sanitari de L'Alt Penedes I Garraf, Barcelona, Spain.
Received: 15 June 2020 Accepted: 4 August 2020

Published online: 14 August 2020

\section{References}

1. Mehra MR, Canter CE, Hannan MM, et al. The 2016 International Society for Heart Lung Transplantation listing criteria for heart transplantation: a 10-year update. J Heart Lung Transpl. 2016;35(1):1-23.

2. Jaiswal A, Truby LK, Chichra A, et al. Impact of obesity on ventricular assist device outcomes. J Card Fail. 2020;26(4):287-97.

3. Leviner DB, Keidar A, Ben-Gal T, et al. Cardiac function recovery following LVAD implantation and bariatric surgery in a morbidly obese patient. J Card Surg. 2014;29(5):740-2.

4. Morrow EH, Pellegrini CA, Mokadam NA, et al. Laparoscopic gastric bypass during left ventricular assist device support and ventricular recovery. J Heart Lung Transplant. 2014;33(8):870-1.

5. Zenilman A, Pechman D, Moran-Atkin E, et al. Bariatric surgery in patients with left ventricular assist devices: a safe and effective method of weight loss as a gateway to heart transplantation. Surg Obes Relat Dis. 2019;15(10):1780-4.

6. Punchai S, Nor Hanipah Z, Sharma G, et al. Laparoscopic sleeve gastrectomy in heart failure patients with left ventricular assist device. Obes Surg. 2019;29(4):1122-9.

7. Uriel N, Colombo PC, Cleveland JC, et al. Hemocompatibility-related outcomes in the MOMENTUM 3 Trial at 6 months: a randomized controlled study of a fully magnetically levitated pump in advanced heart failure. Circulation. 2017:135(21):2003-122.

8. daSilva-deAbreu A, Alhafez BA, Curbelo-Pena Y, et al. Bariatric surgery in obese patients with ventricular assist devices considered for heart transplantation: systematic review and individual participant data metaanalysis. In peer-review.

9. Lockard KL, Allen C, Lohmann D, et al. Bariatric surgery for a patient with a HeartMate II ventricular assist device for destination therapy. Progr Transpl. 2013;23(1):28-322.

10. Wikiel KJ, McCloskey CA, Ramanathan RC. Bariatric surgery: a safe and effective conduit to cardiac transplantation. Surg Obes Relat Dis. 2014;10(3):479-84.

11. daSilva-deAbreu A, et al. Bariatric surgery in obese patients with endstage heart failure with ventricular assist devices. Mendeley. 2020. https:// doi.org/10.17632/33m7sc3wnc.2.

\section{Publisher's Note}

Springer Nature remains neutral with regard to jurisdictional claims in published maps and institutional affiliations. 